\title{
Comparative Economic Outcomes in Patients with Focal Seizure Initiating First-Line Eslicarbazepine Acetate Monotherapy versus Generic Antiseizure
} Drugs

\author{
Darshan Mehta $\mathbb{D}^{\prime}$ \\ Matthew Davis $\mathbb{D}^{2}$ \\ Andrew J Epstein (1D ${ }^{3}$ \\ Brian Wensel' \\ Todd Grinnell ${ }^{4}$ \\ G Rhys Williams' \\ 'Sunovion Pharmaceuticals Inc., \\ Marlborough, MA, USA; ${ }^{2}$ Medicus \\ Economics, LLC, Milton, MA, USA; \\ ${ }^{3}$ Medicus Economics, LLC, Philadelphia, \\ PA, USA; ${ }^{4}$ Medical Affairs, Sunovion \\ Pharmaceuticals Inc., Marlborough, \\ MA, USA
}

Objective: To examine the association between initiating first-line (1L) monotherapy with eslicarbazepine acetate (ESL) vs a generic antiseizure drug (ASD) and healthcare resource utilization (HCRU) and charges in adults with treated focal seizures (FS).

Methods: This was a retrospective analysis of Symphony Health's Integrated Dataverse ${ }^{\circledR}$ open-source claims data. Two cohorts were identified as having initiated 1L monotherapy with ESL or literature-defined generic ASDs. Linear regression models with person fixed effects and inverse probability treatment weights assessed the relative additional changes in HCRU and charges among patients who received ESL compared to generic ASD.

Results: A total of 250 and 43,220 patients initiated ESL (48.3 years; 57.2\% female) or a generic ASD (54.5 years; $58.1 \%$ female), respectively. Compared to patients initiating a generic ASD, patients treated with ESL had additional reductions of 11.8 percentage points in the likelihood of any all-cause outpatient visits $(P<0.001), 7.4$ percentage points in the likelihood of any emergency department (ED) visits $(P=0.013)$, and 22.7 percentage points in the likelihood of any FS-related outpatient visits $(P<0.001)$. Patients initiating ESL had greater reductions in mean charges for all-cause medical $(\$ 2620 ; P=0.002)$, outpatient ( $\$ 1995 ; P=0.005)$, and non-FS-related medical $(\$ 2708 ; P<0.001)$ services. Patients initiating ESL had greater relative increases in mean total prescription $(\$ 1368 ; P<0.001)$ and ASDrelated prescription $(\$ 1636 ; P<0.001)$ charges, but greater relative reductions in non-ASD prescription ( $\$ 269 ; P=0.032$ ) charges. The increases in prescription charges were of a lower magnitude than the decreases in medical charges.

Conclusion: Initiation of ESL as 1L monotherapy was associated with statistically significantly greater reductions in any use of several all-cause and FS-related services, number of visits, and charges compared to initiation of a generic ASD as 1L monotherapy in patients with FS. Initiation of a generic ASD as 1L monotherapy was associated with significantly smaller increases in total prescription charges and ASDrelated prescription charges.

Keywords: eslicarbazepine acetate, focal seizures, healthcare resource utilization, medical charges

\section{Background}

In the United States (US), the estimated prevalence of active epilepsy is $1.2 \%$, including approximately 3 million adults aged 18 years or older. ${ }^{1}$ Focal seizures (FS) constitute an estimated $60 \%$ of epilepsy cases. $^{2-5}$ 
Multiple antiseizure drugs (ASDs), classified as first- (phenytoin, valproate, carbamazepine [CBZ], clobazam), second(gabapentin, lacosamide, lamotrigine [LTG], levetiracetam [LEV], oxcarbazepine, rufinamide, tiagabine, topiramate, vigabatrin, zonisamide), or third-generation (eslicarbazepine acetate [ESL], brivaracetam, perampanel ${ }^{3,6-8}$ drugs, are available to treat epilepsy. LEV, a second-generation ASD, is the firstline (1L) standard of care ASD therapy in the US for FS. ${ }^{9,10}$ Third-generation ASDs are typically used in later lines of therapy for refractory FS, ${ }^{11}$ but may be applicable earlier in treatment due to better tolerability, milder adverse effects, fewer drug interactions, and improved pharmacokinetic characteristics compared to first- or second-generation ASDs. ${ }^{6}$

ESL, a third-generation ASD, is approved by the US Food and Drug Administration (FDA) for the treatment of FS in adult patients and pediatric patients ages 4 years and older. ${ }^{12}$ Clinical efficacy and safety of ESL as an adjunctive therapy or monotherapy for FS have been demonstrated in several pivotal trials. ${ }^{13-16}$ In a real-world clinical setting, an open-label, Phase IV study demonstrated efficacy and tolerability of ESL in FS as first adjunctive therapy with LEV or LTG. ${ }^{17,18}$

ESL may also have clinical benefits in the 1L setting. Treatment with sodium channel-blocking ASDs, a class of which ESL is a member, ${ }^{11}$ improved 6-month seizure freedom and achieved lower rates of an addition of or a switch to a second ASD compared to LEV in newly treated patients with FS. ${ }^{19}$ Once-daily ESL was non-inferior to twice-daily, controlled-release CBZ monotherapy for seizure-freedom rates in newly diagnosed patients with FS over a 1-year treatment duration. ${ }^{20}$ Use of ESL as initial monotherapy, switching to ESL from CBZ or oxcarbazepine, or conversion to ESL monotherapy after $\geq 1$ ASD, was associated with a $\geq 50 \%$ reduction in seizure frequency in $82.0 \%, 79.7 \%$, and $83.0 \%$ of the patients at 3,6 , and 12 months of follow-up, respectively. ${ }^{21}$

Epilepsy incurs substantial healthcare resource utilization (HCRU) and costs. ${ }^{3,22}$ Direct and indirect costs are higher for patients with epilepsy than controls without the disease. ${ }^{23-25}$ Epilepsy-related direct annual costs are higher in patients with treatment-refractory or uncontrolled epilepsy compared to patients who are treatmentresponsive and stable. ${ }^{26}$

Prior literature demonstrates better outcomes among patients who initiated second- or third-generation ASDs relative to those who initiated first-generation ASDs. In adults with epilepsy or those who lacked an epilepsy diagnosis but received $\geq 1$ prescription for an FDA-approved ASD, second- generation ASDs reduced the frequency of epilepsy-related hospitalizations compared to first-generation ASDs. ${ }^{27}$ In adults with FS, all-cause and FS-related HCRU and charges were significantly reduced following initiation of ESL monotherapy both as $1 \mathrm{~L}$ treatment or as first adjunctive regimen. ${ }^{28}$ However, data comparing economic outcomes following initiation of third-generation ASDs as 1L treatment vs standard of care (ie, generic ASDs) are lacking. The objective of the present study was to study the association between starting 1L monotherapy with ESL vs a generic ASD on HCRU and charges among adult patients with treated FS.

\section{Methods Data Source}

The analysis used administrative claims data extracted from Symphony Health's Integrated Dataverse (IDV ${ }^{\mathbb{B}}$; hereafter referred to as the database), which is a national open-source database of pharmacy claims, physician office medical claims, and hospital claims across a broad range of payer types, including commercial, Medicare, Medicaid, and self-pay/uninsured. The database contains information on an estimated 274 million active patients, including longitudinal data on medical resource use, diagnoses, procedures, prescription fills, and charges for visits to covered providers. The data are de-identified in compliance with the Health Insurance Portability and Accountability Act. Therefore, this study did not constitute Human Subjects Research, ${ }^{29}$ and review by an institutional review board was not required.

\section{Study Design}

This retrospective, longitudinal cohort study compared a cohort of patients with FS who initiated ESL as 1L monotherapy to a cohort of patients with FS who initiated a generic ASD as 1L monotherapy. Index date was the date of their earliest claim for ESL or a generic ASD. The study period was April 1, 2015, to June 30, 2018. A longitudinal panel data approach was used, with a person-specific period of 90 consecutive days as the unit of analysis. The baseline period was defined as the 90-day period immediately prior to the index date. The follow-up period was defined as at least one and up to four consecutive 90-day periods immediately following the index date. Changes in HCRU and charges from 90 days before to up to 360 days after 1L monotherapy initiation were compared between patients on ESL vs a generic ASD (Figure 1). 


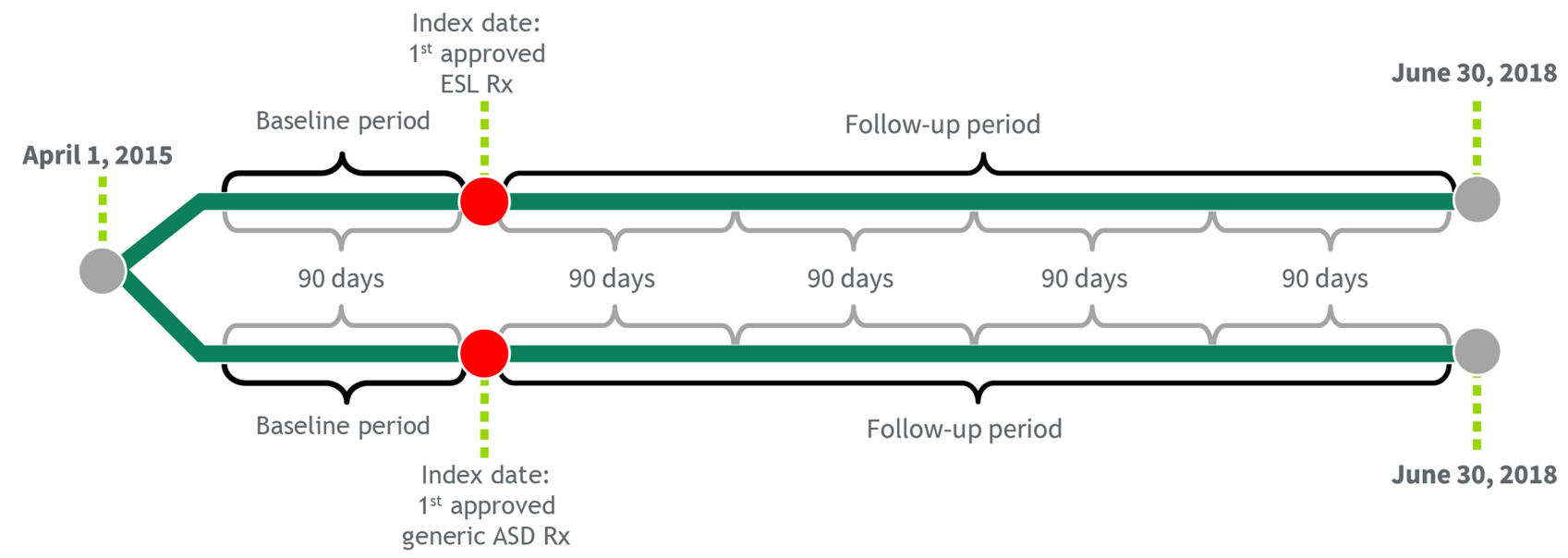

Figure I Study design.

Abbreviations: ASD, antiseizure drug; ESL, eslicarbazepine acetate; Rx, prescription.

\section{Study Population}

This study included patients with treated FS with no evidence of ASD utilization prior to their index date. Patients qualified for one of two mutually exclusive cohorts: those who initiated ESL as 1L monotherapy and those who initiated a generic ASD as 1L monotherapy. The generic ASD cohort comprised patients with $\geq 1$ dispensed pharmacy claim for the 1L ASDs most commonly used in clinical practice in the US, as reported in the published literature. These ASDs were identified in a retrospective database analysis using a data-driven approach and included LEV, phenytoin, valproic acid, LTG, oxcarbazepine, topiramate, gabapentin, ethosuximide, phenobarbital, and clonazepam. ${ }^{10}$

Other key sample selection criteria were: 1) US residence; 2) $\geq 1$ one medical claim with an FS diagnosis code (International Classification of Diseases, Ninth Revision, Clinical Modification [ICD-9-CM]) diagnosis codes $345.4 \mathrm{x}$ or $345.5 \mathrm{x}$ or ICD-10-CM diagnosis codes G40.1x or $\mathrm{G} 40.2 \mathrm{x}$ ); 3) no claim for an ASD prior to their index date; 4) index ASD (ESL or a generic ASD) was a new prescription; 5) no other ASD claim on the index date; 6) $\geq 180$ days between the start of data coverage (April 1, 2015) and the index date; 7) $\geq 18$ years old on the index date (assuming a birth date of July 1 because only birth year was available); 8) $\geq 1$ medical claim with an FS diagnosis before or within 360 days after their index date; 9) $\geq 1$ medical claim and at least one prescription drug claim during the 180 days prior to the index date; 10) no diagnoses indicating active pregnancy (ICD-9-CM codes: V22.x, V23.xx, 630-679.xx; ICD-10-CM codes:
$\mathrm{Z} 33 *-\mathrm{Z} 36 * \mathrm{O} 00 *-\mathrm{O} 99 *)$ during the 180 days prior to the index date; 11) $\geq 1$ medical claim and $\geq 1$ prescription drug claim at any time following the index date; and 12) $\geq 90$ days between the index date and the end of data coverage (June 30, 2018).

\section{Study Measures \\ Baseline Characteristics}

Demographic characteristics were measured during the baseline period and included age, gender, and region (Midwest, Northeast, South, West).

Clinical characteristics included comorbidities and ASD-related characteristics. Comorbidities were measured during the baseline period based on the presence of at least one medical claim with a diagnosis code for alcohol/drug dependence, anoxic brain injury, atherosclerosis, bipolar disorder, brain tumor, cerebrovascular disease, central nervous system infections, chronic obstructive pulmonary disease, cognitive impairment, congestive heart failure, dementia, diabetes with and without complications, drug dependence, falls and fractures, hemiplegia/paraplegia, hypertension, hyponatremia, intellectual disabilities, major depressive disorder, mild liver disease, myocardial infarction, nervous system neoplasms, Parkinson disease, peptic ulcer disease, peripheral vascular disease, pregnancy, rheumatic disease, schizophrenia, sleep apnea, and traumatic brain injury (diagnosis codes are available from the authors on request).

ASD-related characteristics were measured on the index date and included patient copay, payer type (commercial, Medicaid, Medicare, other), and calendar year of index date. 


\section{HCRU and Charges in the Baseline and Follow-Up Periods}

All-cause and FS-related inpatient, emergency department (ED), outpatient hospital, and office visits were measured for each patient-block during the baseline and follow-up periods. Claims were categorized by place of service. Each HCRU category was presented as the proportion of patients with $\geq 1$ HCRU visit of each type. The number of visits for each type was also studied.

Charges for medical claims, ASD-related and nonASD-related prescription drug charges, totals for allcause medical charges, FS-related medical charges, prescription drug charges, and an overall total were measured for each patient-block during the baseline and follow-up periods.

All-cause HCRU and charges were calculated using all claims, regardless of associated diagnosis codes. FSrelated HCRU and charges were calculated using claims with a diagnosis of FS in any diagnosis position. All dollar-denominated variables were inflated to 2018 US dollars using the US Gross Domestic Product price index to account for general inflation. ${ }^{30,31}$

\section{Statistical Analysis}

For baseline demographic and clinical characteristics, continuous variables were expressed as means and standard deviations (SDs). Dichotomous and categorical variables were expressed as counts and percentages. To assess differences between cohorts at baseline, means were compared with t-tests and percentage distributions were compared with chi-square tests.

Changes in HCRU and charge outcomes from before to after initiation of a 1L ASD monotherapy were compared between individuals who initiated ESL or a generic ASD using a difference-in-differences framework. Both unadjusted and adjusted analyses were conducted. A separate model was run for each of the all-cause and FS-related HCRU and charge categories.

In the unadjusted model specification, a linear regression was estimated to predict each outcome as a function of the exposure (ESL vs generic ASD), an indicator for whether the period was from before or after ASD initiation (pre vs post), and their interaction. The fitted coefficient on the interaction term was interpreted as the relative additional difference between patients who received ESL compared to a generic ASD in the pre-post change in the outcome.
The adjusted model specification extended the unadjusted specification in two ways. First, time-invariant covariates were controlled for by adding person-specific fixed effects, which isolated the identification of the association between ASD initiation and outcomes to within-person changes over time. Second, baseline covariates were balanced between exposure groups using propensity scorebased weighting. ${ }^{32}$ Balance between cohorts for each covariate was assessed via the standardized difference with and without weighting by the odds of receiving ESL. ${ }^{33}$ In the adjusted model specification, observations were weighted by the odds of receiving ESL. The fitted coefficients from the model were interpreted as the relative additional change in patients who received ESL compared to a generic ASD in the outcome.

Regression standard errors were adjusted to be robust to heteroskedasticity and to account for multiple 90-day periods per individual. ${ }^{34}$

Statistical significance was based on two-sided tests with $\alpha=0.05$. Statistical analyses were conducted using SAS software version 9.4 (SAS Institute, Cary, NC) and Stata MP software version 16.1 (StataCorp, LLC, College Station TX).

\section{Results}

\section{Baseline and Clinical Characteristics}

A total of 250 patients who initiated ESL as 1L monotherapy and 43,220 patients who initiated a generic ASD as $1 \mathrm{~L}$ monotherapy met the study inclusion criteria (Figure 2). Twenty-two percent of the patients who initiated ESL and $21 \%$ of the patients who initiated a generic ASD had fewer than four 90-day periods during the follow-up.

The baseline demographic and clinical characteristics of the patients who initiated ESL or a generic ASD are described in Table 1. Patients who initiated ESL were significantly younger (mean [SD] 48.3 [16.3] vs 54.5 [17.9] years; $P<0.001$ ), more frequently from the South (51.6\% vs $39.7 \% ; P<0.001)$, and more frequently covered by commercial insurance $(60.0 \%$ vs $45.2 \% ; P<0.001)$ compared to patients who initiated a generic ASD. Patients who initiated ESL were less likely to experience brain tumors $(0.8 \%$ vs $3.3 \% ; P=0.027)$, congestive heart failure $(2.8 \%$ vs $5.8 \% ; P=0.045)$, cognitive impairment (4.0\% vs $12.2 \% ; P<0.001)$, chronic pulmonary disease $(5.2 \%$ vs $10.7 \% ; \quad P=0.005)$, cerebrovascular disease $(12.8 \%$ vs $24.0 \% ; P<0.001)$, uncomplicated diabetes $(8.0 \%$ vs $14.2 \% ; P=0.005)$, hypertension $(18.4 \%$ vs 


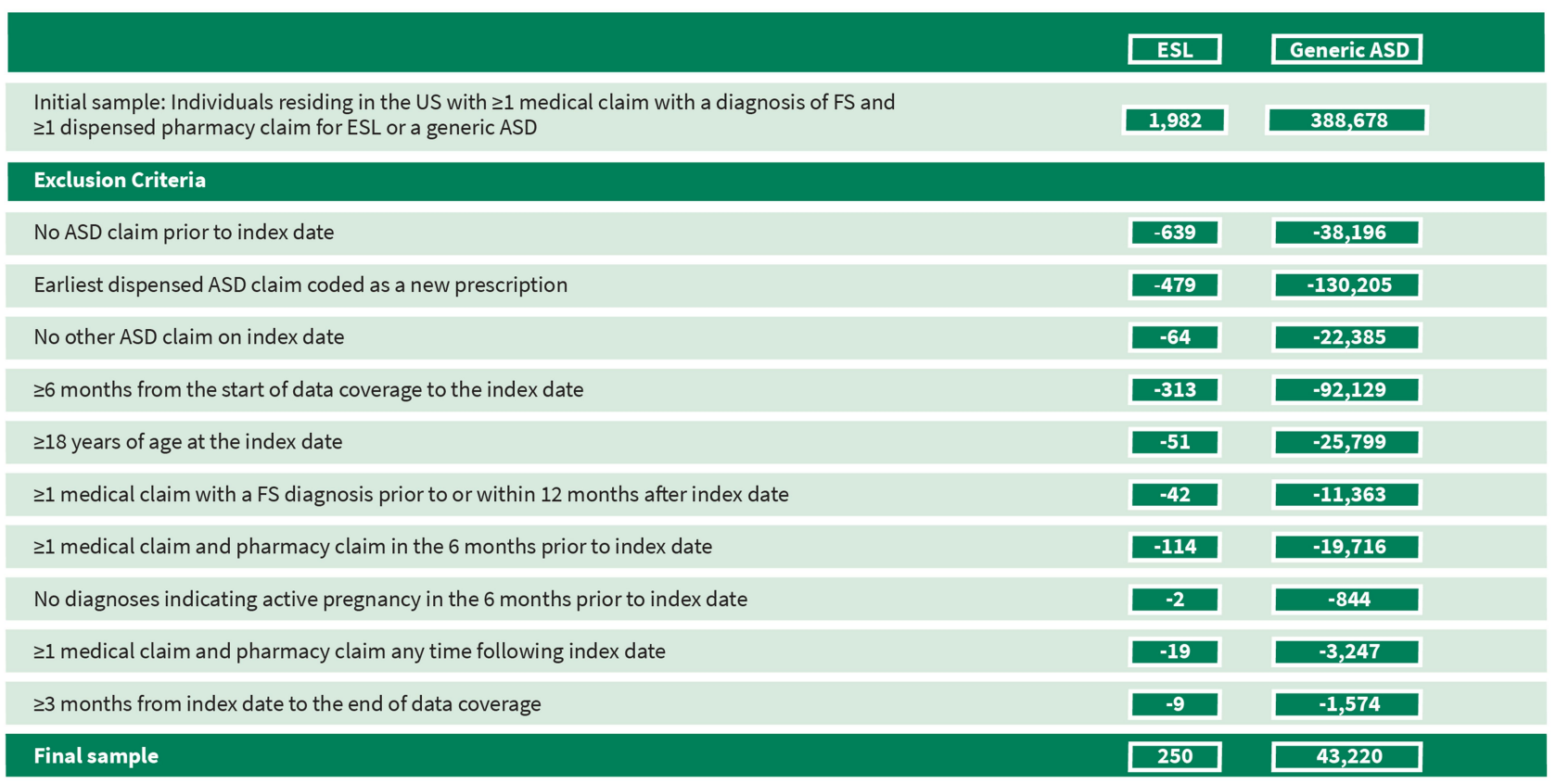

Figure 2 Sample selection.

Abbreviations: ASD, antiseizure drug; ESL, eslicarbazepine acetate; FS, focal seizure; US, United States.

$34.8 \% ; P<0.001)$, hemiplegia/paraplegia $(1.2 \%$ vs $4.8 \%$; $P=0.008)$, peripheral vascular disease $(2.0 \%$ vs $4.7 \%$; $P=0.047)$, or renal disease $(1.2 \%$ vs $6.1 \% ; P=0.001)$ compared to patients who initiated a generic ASD. Among patients who initiated a generic ASD, LEV was the most frequently utilized 1L therapy (55\%), followed by gabapentin $(16 \%)$, LTG $(9.1 \%)$, and topiramate $(8.1 \%)$.

After weighting, all standardized differences between patients who initiated ESL or a generic ASD were within acceptable limits (Supplementary Table 2) ${ }^{35}$

\section{HCRU and Associated Charges HCRU}

Results from the unadjusted linear regression model are presented in Supplementary Table 1. Initiation of ESL as 1L monotherapy was associated with significantly larger unadjusted reductions per 90-day period in any use of allcause outpatient services and FS-related outpatient services compared to initiation of a generic ASD as 1L monotherapy, and significantly larger reductions in number of days of all-cause outpatient services and FS-related outpatient services. Initiation of a generic ASD as 1L monotherapy was associated with significantly larger unadjusted reductions per 90-day period in any use of allcause inpatient hospitalization and FS-related inpatient hospitalization and ED services compared to initiation of
ESL as 1L monotherapy, and larger reductions in number of days of all-cause inpatient hospitalization and FSrelated inpatient hospitalization and ED services.

The difference-in-differences results from the adjusted linear regression model are consistent with the unadjusted results and are shown in Figure 3 and described in Table 2. Initiation of ESL as 1L monotherapy was associated with significantly larger adjusted reductions per 90-day period in any use of all-cause ED services ( -7.4 ppts; $P=0.013$ ) and outpatient services $(-11.8$ ppts; $P<0.001)$ and FSrelated outpatient services $(-22.7$ ppts; $P<0.001)$ compared to initiation of a generic ASD as 1L monotherapy. In addition, there were larger reductions in number of days of all-cause inpatient hospitalization $(-0.25$ days; $P=0.019)$ and outpatient services $(-0.91$ days; $P<0.001)$ and FS-related inpatient hospitalization $(-0.20$ days; $P=0.018)$ and outpatient services ( -0.39 days; $P<0.001)$.

\section{Charges}

Results from the unadjusted linear regression model are presented in Supplementary Table 1. Initiation of ESL as 1L monotherapy was associated with significantly larger unadjusted reductions in all-cause outpatient service charges and non-ASD-related prescription charges. Initiation of a generic ASD as 1L monotherapy was associated with significantly larger unadjusted reductions in all-cause inpatient hospitalization charges and FS-related 
Table I Baseline Demographic and Clinical Characteristics for Patients Receiving ESL and Patients Receiving Generic ASD as IL Therapy

\begin{tabular}{|c|c|c|c|}
\hline Variable & ESL $(\mathbf{N}=\mathbf{2 5 0})$ & Generic ASD $(N=43,220)$ & $P$-value \\
\hline Patient age (years), mean (SD) & $48.3(16.3)$ & $54.5(17.9)$ & $<0.001$ \\
\hline Patient age category, n (\%) & & & $<0.001$ \\
\hline $18-39$ & $87(34.8)$ & $10,615(24.6)$ & \\
\hline $40-64$ & $119(47.6)$ & I7,872 (4|.4) & \\
\hline $65+$ & $44(17.6)$ & $14,733(34.1)$ & \\
\hline Patient gender, n (\%) & & & 0.77 \\
\hline Male & $107(42.8)$ & |8,097 (4|.9) & \\
\hline Female & $143(57.2)$ & $25,123(58.1)$ & \\
\hline Geographic region, n (\%) & & & $<0.001$ \\
\hline Midwest & $45(18.0)$ & $10,595(24.5)$ & \\
\hline Northeast & $52(20.8)$ & $9594(22.2)$ & \\
\hline South & $129(51.6)$ & $17,153(39.7)$ & \\
\hline West & $24(9.6)$ & $5878(13.6)$ & \\
\hline Rx payer, n (\%) & & & $<0.001$ \\
\hline Commercial & $150(60.0)$ & $19,549(45.2)$ & \\
\hline Medicaid & $39(15.6)$ & $8403(19.4)$ & \\
\hline Medicare & $43(17.2)$ & 13,433 (31.1) & \\
\hline Other & $18(7.2)$ & $1835(4.3)$ & \\
\hline Patient copay amount ${ }^{\mathrm{a}, \mathrm{b}}$, mean (SD) & $\$ 82(177)$ & $\$ 12(60)$ & $<0.001$ \\
\hline Year of index date, n (\%) & & & 0.54 \\
\hline 2015 & $38(15.2)$ & $5947(13.8)$ & \\
\hline 2016 & III (44.4) & $19,695(45.6)$ & \\
\hline 2017 & $81(32.4)$ & $14,922(34.5)$ & \\
\hline 2018 & $20(8.0)$ & $2656(6.2)$ & \\
\hline \multicolumn{4}{|l|}{ Comorbidities $^{c}$, n (\%) } \\
\hline Hypertension & $46(18.4)$ & $15,019(34.8)$ & $<0.001$ \\
\hline Cerebrovascular disease & $32(12.8)$ & $10,356(24.0)$ & $<0.001$ \\
\hline Diabetes without chronic complication & $20(8.0)$ & $6136(14.2)$ & 0.005 \\
\hline Cognitive impairment & $10(4.0)$ & $5268(12.2)$ & $<0.001$ \\
\hline Bipolar disorder & $26(10.4)$ & $4769(11.0)$ & 0.75 \\
\hline Chronic pulmonary disease & $13(5.2)$ & $4625(10.7)$ & 0.005 \\
\hline Major depressive disorder & $22(8.8)$ & $3892(9.0)$ & 0.91 \\
\hline Sleep apnea & $19(7.6)$ & $2457(5.7)$ & 0.19 \\
\hline Central nervous system infections & II (4.4) & $3283(7.6)$ & 0.057 \\
\hline Falls and fractures & $12(4.8)$ & $3100(7.2)$ & 0.15 \\
\hline Nervous system neoplasms & $9(3.6)$ & $2617(6.1)$ & 0.10 \\
\hline Renal disease & $3(1.2)$ & $2656(6.1)$ & 0.001 \\
\hline Congestive heart failure & $7(2.8)$ & $2488(5.8)$ & 0.045 \\
\hline Traumatic brain injury & $12(4.8)$ & $1434(5.6)$ & 0.57 \\
\hline IL therapy, n (\%) & & & $<0.001$ \\
\hline ESL & $250(100)$ & $0(0)$ & \\
\hline LEV & $0(0)$ & $23,861(55)$ & \\
\hline Gabapentin & $0(0)$ & $6910(16)$ & \\
\hline LTG & $0(0)$ & $3928(9.1)$ & \\
\hline Topiramate & $0(0)$ & $3500(8.1)$ & \\
\hline Phenytoin & $0(0)$ & 1673 (3.9) & \\
\hline Oxcarbazepine & $0(0)$ & $1668(3.9)$ & \\
\hline
\end{tabular}


Table I (Continued).

\begin{tabular}{|l|c|c|c|}
\hline Variable & ESL (N=250) & Generic ASD (N=43,220) & P-value \\
\hline Clonazepam & $0(0)$ & $1303(3)$ & \\
Valproic acid & $0(0)$ & $222(0.5)$ & $145(0.3)$ \\
Phenobarbital & $0(0)$ & $10(0.0)$ & \\
Ethosuximide & $0(0)$ & \\
\hline
\end{tabular}

Notes: ${ }^{a}$ Value taken from index claim; ${ }^{\mathrm{b}}$ Dollar values of charges inflated to 2018 US dollars using the US GDP price index; ${ }^{\mathrm{c}}$ Comorbidities with a prevalence of $<5 \%$ : anoxic brain injury, alcohol/drug dependence, brain tumor, dementia, intellectual disability, diabetes with chronic complication, mild liver disease, myocardial infarction, hyponatremia, Parkinson's disease, hemiplegia or paraplegia, peptic ulcer disease, peripheral vascular disease, rheumatic disease, schizophrenia.

Abbreviations: IL, first-line; ASD, antiseizure drug; ESL, eslicarbazepine acetate; GDP, gross domestic product; LEV, levetiracetam; LTG, lamotrigine; Rx, prescription; SD, standard deviation; US, United States.

ED charges and smaller increases in total prescription charges and ASD-related prescription charges.

In adjusted analyses (Table 2, Figure 3), initiation of ESL as $1 \mathrm{~L}$ monotherapy was associated with significantly larger adjusted reductions in all-cause medical charges $(-\$ 2620$; $P=0.002)$, all-cause outpatient charges $(-\$ 1995 ; P=0.005)$, non-FS-related medical charges $(-\$ 2708 ; P<0.001)$, and non-ASD-related prescription charges $(-\$ 269 ; P=0.032)$. Initiation of a generic ASD as 1L monotherapy was associated with significantly smaller adjusted increases in total prescription charges $(\$ 1368 ; P<0.001)$ and ASD-related prescription charges $(\$ 1636 ; P<0.001)$ (Figure 3; Table 2).

\section{Discussion}

Findings from this study suggest there was a beneficial economic impact associated with initiating $1 \mathrm{~L}$ monotherapy with ESL compared to a generic ASD among adult patients with treated FS, as demonstrated by significantly larger relative additional reductions in HCRU and charges associated with specific claim types. These included any use of all-cause ED and outpatient services and FS-related outpatient services, number of days of all-cause and FS-related inpatient hospitalization and outpatient services, and all-cause medical charges and non-ASD-related prescription charges. The increases in prescription charges were of lower magnitude as compared to the decrease in total medical charges.

The economic benefits associated with initiating ESL as $1 \mathrm{~L}$ therapy in the present study may be driven by the efficacy of ESL in the real world. In the Human Epilepsy Project, a prospective multicenter study, drugs with a similar mechanism of action had improved 6-month (61\% vs 37\%) and terminal seizure freedom ( $52 \%$ vs $30 \%$ ) in patients with newly treated FS compared to LEV monotherapy. ${ }^{19}$ In a multicenter observational study, 87.0\%, 78.5\%, and $75.0 \%$ of the patients with FS using ESL as initial monotherapy obtained a $\geq 50 \%$ reduction in seizure frequency, and
$77.4 \%, 75.0 \%$, and $68.7 \%$ were seizure-free at 3,6 , and 12 months of follow-up, respectively. ${ }^{21}$ Further research is required to fully understand the role of ESL in alleviating the economic burden of epilepsy in clinical practice.

The burden on HCRU and costs posed by epilepsy is well established. ${ }^{3,22-26}$ Most forms of epilepsy require lifelong therapy to control seizures; therefore, choice of treatment should include economic considerations. ${ }^{36}$ Some evidence suggests that the use of second-generation ASDs is associated with lower rates of HCRU compared to the use of firstgeneration ASDs. A retrospective claims-based study in patients aged $\geq 12$ years with epilepsy or one or more prescriptions for ASDs with epilepsy as the only FDA-approved indication showed that patients taking second-generation ASDs (LEV, LTG, topiramate, gabapentin) experienced fewer epilepsy-related hospitalizations compared to patients taking first-generation ASDs (phenytoin, CBZ, valproic acid) (one epilepsy-related hospitalization every 1001 days vs 684 days; relative risk reduction of $31 \%, P<0.01) .{ }^{27} \mathrm{HCRU}$ and healthcare costs may be further decreased by the use of ESL, a third-generation ASD, as 1L therapy. In a previous study in patients with FS who initiated 1L ESL, there were significant reductions in all-cause inpatient, ED, and outpatient visits; FS-related inpatient and outpatient visits; and total, medical, all-cause ED and outpatient, and FS-related medical charges during the follow-up period. ${ }^{28}$ The present study extends these findings by showing reduced HCRU and costs in patients with FS initiating ESL as 1L monotherapy compared to the most widely used first- or second-generation ASD as $1 \mathrm{~L}$ agent.

A retrospective cohort study that included commercial, supplemental Medicare, and Medicaid insurance claims from the US-based Truven Health MarketScan ${ }^{\circledR}$ claims database demonstrated that LEV is the most commonly prescribed 1L ASD in the US. ${ }^{10}$ This is likely because physicians are aware of the safety and efficacy of LEV as 
A $\quad 15$

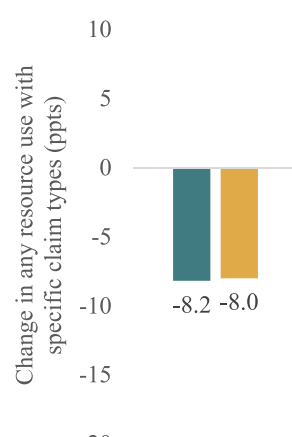

$-20$

$-25$
- ESL - Generic ASD

FS-related

inpatient hospitalization
FS-related ED FS-related outpatient

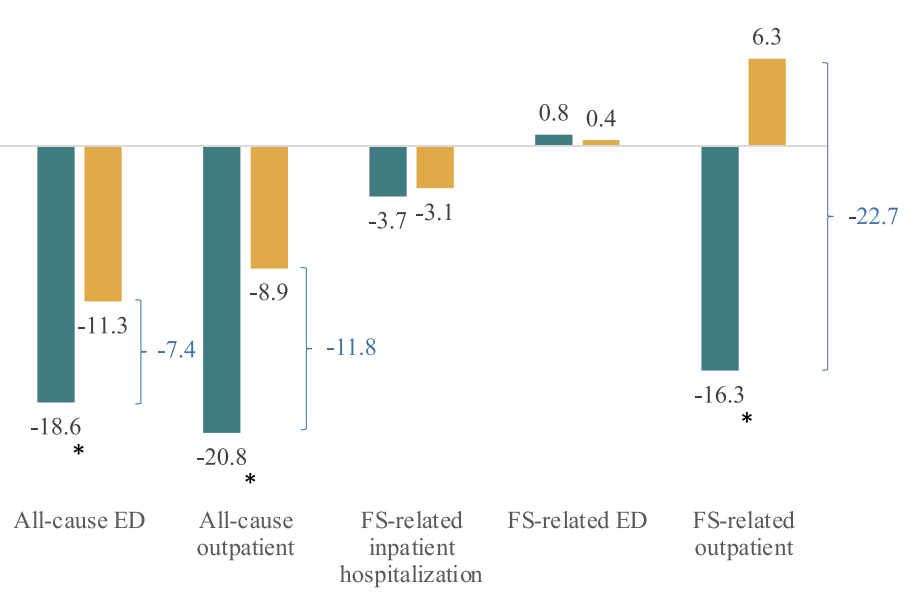

B

- ESL Generic ASD

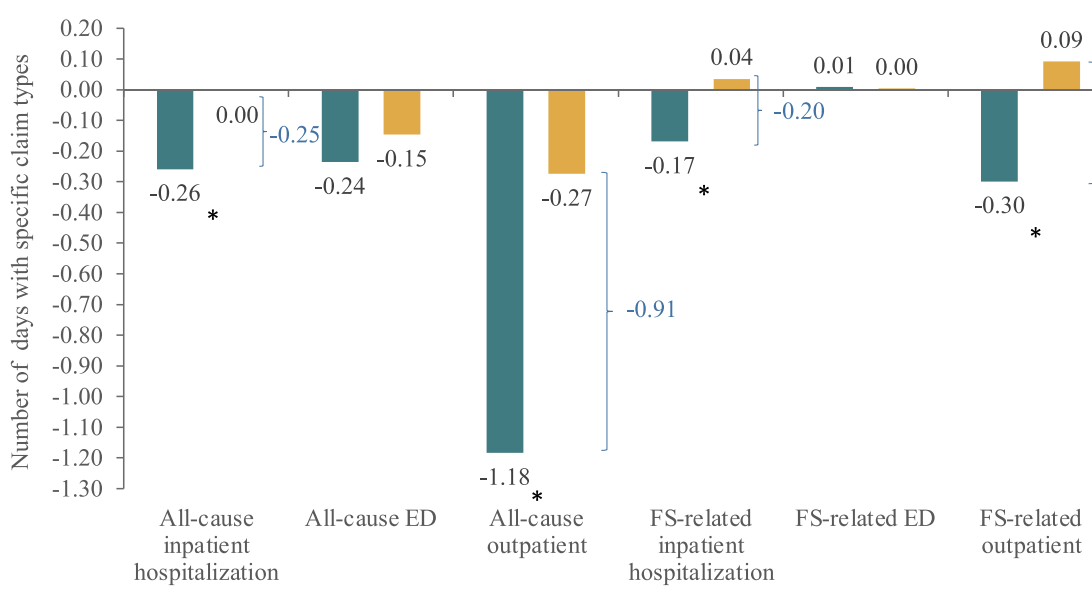

C

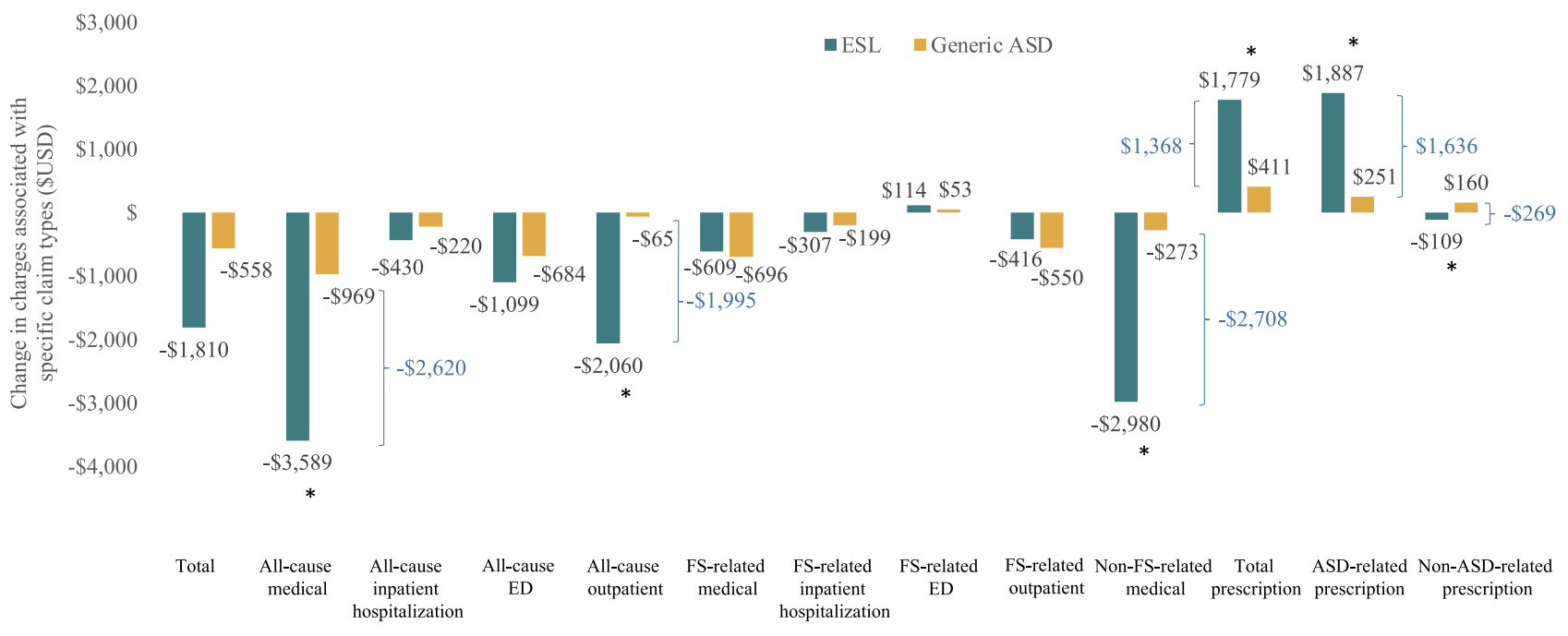

Figure 3 Relative additional change in any HCRU (A), number of days of HCRU (B), or charges (C) for patients initiating ESL as monotherapy compared to a generic ASD. All outcomes were measured over 90 day periods. (A) Any HCRU. (B) Number of days of HCRU. (C) Charges. $*$ P<0.05 ESL vs generic ASD.

Abbreviations: ASD, antiseizure drug; ED, emergency department; ESL, eslicarbazepine acetate; FS, focal seizure; HCRU, healthcare resource utilization; Ppts, percentage points. 
Table 2 Adjusted Difference-in-Differences of Resource Use and Cost Outcomes

\begin{tabular}{|c|c|c|c|c|c|c|}
\hline \multirow[b]{2}{*}{ Outcome Name } & \multicolumn{2}{|c|}{ Pre-Post Difference } & \multirow[b]{2}{*}{$\begin{array}{c}\text { ESL-Generic } \\
\text { DD }\end{array}$} & \multirow[b]{2}{*}{$P$-value } & \multirow[b]{2}{*}{$\begin{array}{l}\text { Lower } \\
95 \% \text { CL }\end{array}$} & \multirow[b]{2}{*}{$\begin{array}{l}\text { Upper } \\
95 \% \mathrm{CL}\end{array}$} \\
\hline & $\begin{array}{c}\text { Generic } \\
\text { ASD }\end{array}$ & ESL & & & & \\
\hline \multicolumn{7}{|l|}{ Any resource use with specific claim types ${ }^{a}$} \\
\hline Any use of all-cause inpatient hospitalization & -0.080 & -0.082 & -0.002 & 0.91 & -0.047 & 0.042 \\
\hline Any use of all-cause ED & -0.113 & -0.186 & -0.074 & 0.013 & -0.132 & -0.016 \\
\hline Any use of all-cause outpatient & -0.089 & -0.208 & -0.118 & $<0.001$ & -0.164 & -0.072 \\
\hline Any use of FS-related inpatient hospitalization & -0.031 & -0.037 & -0.006 & 0.67 & -0.033 & 0.021 \\
\hline Any use of FS-related ED & 0.004 & 0.008 & 0.004 & 0.27 & -0.003 & 0.010 \\
\hline Any use of FS-related outpatient & 0.063 & -0.163 & -0.227 & $<0.001$ & -0.295 & -0.158 \\
\hline \multicolumn{7}{|l|}{ Number of days with specific claim types } \\
\hline Number of days of all-cause inpatient hospitalization & 0.00 & -0.26 & -0.25 & 0.019 & -0.47 & -0.04 \\
\hline Number of days of all-cause ED & -0.15 & -0.24 & -0.09 & 0.07 & -0.19 & 0.01 \\
\hline Number of days of all-cause outpatient & -0.27 & -1.18 & -0.91 & $<0.001$ & -1.27 & -0.55 \\
\hline Number of days of FS-related inpatient hospitalization & 0.04 & -0.17 & -0.20 & 0.018 & -0.37 & -0.03 \\
\hline Number of days of FS-related ED & 0.00 & 0.01 & 0.00 & 0.25 & 0.00 & 0.01 \\
\hline Number of days of FS-related outpatient & 0.09 & -0.30 & -0.39 & $<0.001$ & -0.52 & -0.26 \\
\hline \multicolumn{7}{|l|}{ Charges associated with specific claim types } \\
\hline Total charges & $-\$ 558$ & $-\$ 1810$ & $-\$ 1252$ & 0.16 & $-\$ 3016$ & $\$ 512$ \\
\hline All-cause medical charges & $-\$ 969$ & $-\$ 3589$ & $-\$ 2620$ & 0.002 & $-\$ 4269$ & $-\$ 971$ \\
\hline All-cause inpatient hospitalization charges & $-\$ 220$ & $-\$ 430$ & $-\$ 210$ & 0.41 & $-\$ 710$ & $\$ 289$ \\
\hline All-cause ED charges & $-\$ 684$ & $-\$ 1099$ & $-\$ 415$ & 0.18 & $-\$ 1015$ & $\$ 186$ \\
\hline All-cause outpatient charges & $-\$ 65$ & $-\$ 2060$ & $-\$ 1995$ & 0.005 & $-\$ 3383$ & $-\$ 607$ \\
\hline FS-related inpatient hospitalization charges & $-\$ 199$ & $-\$ 307$ & $-\$ 108$ & 0.60 & $-\$ 508$ & $\$ 293$ \\
\hline FS-related ED charges & $\$ 53$ & $\$ 114$ & $\$ 62$ & 0.30 & $-\$ 55$ & $\$ 179$ \\
\hline FS-related outpatient charges & $-\$ 550$ & $-\$ 416$ & $\$ 134$ & 0.69 & $-\$ 534$ & $\$ 801$ \\
\hline FS-related medical charges & $-\$ 696$ & $-\$ 609$ & $\$ 88$ & 0.83 & $-\$ 695$ & $\$ 870$ \\
\hline Non-FS-related medical charges & $-\$ 273$ & $-\$ 2980$ & $-\$ 2708$ & $<0.001$ & $-\$ 4179$ & $-\$ 1236$ \\
\hline Total prescription charges & $\$ 411$ & $\$ 1779$ & $\$ 1368$ & $<0.001$ & $\$ 1002$ & $\$ 1733$ \\
\hline ASD-related prescription charges & $\$ 251$ & $\$ 1887$ & $\$ 1636$ & $<0.001$ & $\$ 1378$ & $\$ 1895$ \\
\hline Non-ASD-related prescription charges & $\$ 160$ & $-\$ 109$ & $-\$ 269$ & 0.032 & $-\$ 515$ & $-\$ 22$ \\
\hline
\end{tabular}

Notes: ${ }^{a}$ Pre-post differences, difference-in-differences, and their corresponding confidence levels are interpreted as percentages: $\mathrm{Difference,} \mathrm{DD}$, or $\mathrm{CL} \times \mathrm{I} 00=\mathrm{X} \%$. Abbreviations: ASD, antiseizure drug; CL, confidence limit; DD, difference-in-differences; ED, emergency department; ESL, eslicarbazepine acetate; FS, focal seizure.

1L therapy for treating seizures. ${ }^{27}$ Findings from the present study showing improved HCRU and charges support additional research on ESL as a 1L option.

This study has several limitations. Given its open-source nature, the database may not capture all claims for a given patient, including claims processed through different claims transaction networks or non-electronic prescriptions that were abandoned prior to being submitted to a pharmacy, information about secondary payers, and paid amounts or costs faced by the provider. The database is based on administrative data not designed for research; consequently, data may be missing or miscoded. Identification of clinical conditions is limited to using ICD-9-CM and ICD-10-CM diagnosis codes on individual claims, and detailed clinical data may be missing. Patients in the ESL cohort may have differed from their peers in the generic ASD cohort in ways that influenced outcomes and were not accounted for in this analysis. The results may be inaccurate because of limitations in the database that led to mismeasurement of variables; for example, the data preclude distinguishing between missing claims data and the true absence of medical care use. Relatively small sample sizes may have led to low statistical power and an inability to find statistically significant differences between cohorts. This was an observational study that showed a beneficial economic impact associated with initiating 1L monotherapy with ESL compared to a generic ASD 
among adult patients with treated FS. Evidence from the published literature implies that drivers of this economic benefit may include the efficacy, ${ }^{19,21}$ tolerability, ${ }^{6,37-39}$ and simplified dosing ${ }^{40,41}$ regimen of ESL. Causative factors were not investigated in the present study but could be an area of future research.

\section{Conclusions}

Initiation of ESL as 1L monotherapy was associated with significantly larger reductions in any use of all-cause ED and outpatient and FS-related outpatient services, number of days of all-cause and FS-related inpatient hospitalization and outpatient services, and all-cause medical and outpatient, non-FS-related medical, and non-ASD-related prescription charges compared to initiation of a generic ASD as 1L monotherapy in patients with FS. Initiation of a generic ASD as 1L monotherapy was associated with significantly smaller adjusted increases in total prescription charges and ASD-related prescription charges. The increases in prescription charges were of a lower magnitude than the decreases in medical charges.

\section{Data Sharing Statement}

The datasets generated and/or analyzed during the current study are not publicly available due to a licensing agreement with Symphony Health's Integrated Dataverse ${ }^{\circledR}$.

\section{Compliance with Ethics Guidelines} $\mathrm{IDV}^{\circledR}$ data are de-identified in compliance with the Health Insurance Portability and Accountability Act. Therefore, this study did not constitute Human Subjects Research, and review by an institutional review board was not required.

\section{Acknowledgments}

Medical writing support was provided by Jane Kondejewski, $\mathrm{PhD}$ of SNELL Medical Communication Inc.

\section{Author Contributions}

All authors made substantial contributions to conception and design, acquisition of data, or analysis and interpretation of data; took part in drafting the article or revising it critically for important intellectual content; agreed to submit to the current journal; gave final approval of the version to be published; and agree to be accountable for all aspects of the work.

\section{Funding}

This work was sponsored by Sunovion Pharmaceuticals Inc. Sunovion participated in the study design, analysis and interpretation of data, and review and approval of the manuscript to submit for publication. Funding for manuscript development was provided by Sunovion Pharmaceuticals Inc.

\section{Disclosure}

DM, BW, TG, and GRW are employees of Sunovion Pharmaceuticals Inc. MD and AJE are employees of Medicus Economics, LLC, which received funding from Sunovion Pharmaceuticals Inc. to participate in this research. The authors report no other conflicts of interest in this work.

\section{References}

1. Zack MM, Kobau R. National and state estimates of the numbers of adults and children with active epilepsy - United States, 2015. MMWR Morb Mortal Wkly Rep. 2017;66(31):821-825. doi:10.15 585/mmwr.mm6631a1

2. Banerjee PN, Filippi D, Hauser WA. The descriptive epidemiology of epilepsy- a review. Epilepsy Res. 2009;85(1):31-45. doi:10.1016/j. eplepsyres.2009.03.003

3. Clinical Brief. Examining the economic impact and implications of epilepsy. Am J Manag Care. 2020;1-5.

4. National Institute of Neurological Disorders and Stroke. The epilepsies and seizures: hope through research; 2020. Available from: https://www.ninds.nih.gov/Disorders/Patient-Caregiver-Education /Hope-Through-Research/Epilepsies-and-Seizures-Hope-Through. Accessed December 3, 2020.

5. Wang Z, Li X, Powers A, et al. Outcomes associated with switching from monotherapy to adjunctive therapy for patients with partial onset seizures. Expert Rev Pharmacoecon Outcomes Res. 2015;15 (2):349-355. doi:10.1586/14737167.2015.989217

6. de Biase S, Nilo A, Bernardini A, et al. Timing use of novel anti-epileptic drugs: is earlier better? Expert Rev Neurother. 2019;19(10):945-954. doi:10.1080/14737175.2019.1636649

7. Kanner AM, Ashman E, Gloss D, et al. Practice guideline update summary: efficacy and tolerability of the new antiepileptic drugs I: treatment of new-onset epilepsy. Neurology. 2018;91(2):74-81. doi:10.1212/WNL.0000000000005755

8. Besag FMC, Patsalos PN. New developments in the treatment of partial-onset epilepsy. Neuropsychiatr Dis Treat. 2012;8:455-464.

9. Cassard L, Hegde M, Gidal BE, et al. Levetiracetam versus sodium channel blockers as first prescribed antiepileptic drug: data from the human epilepsy project. American Epilepsy Society Annual Meeting; 6-10 December 2019; Baltimore (MD). Abstract 1.318.

10. Faught E, Helmers SL, Thurman D, et al. Patient characteristics and treatment patterns in patients with newly diagnosed epilepsy: a US database analysis. Epilepsy Behav. 2018;85:37-44. doi:10.1016/j. yebeh.2018.05.019

11. Conway JM, Tallian KB. Epilepsy. In: American College of Clinical Pharmacy, editor. Pharmacology Self-Assessment Program (PSAP) 2018 Book 3 Neurology and Psychiatry. 2018:7-27. Available from:. https://www.accp.com/store/product. aspx?pc=PSAP18_3.

12. Sunovion Pharmaceuticals Inc. APTIOM ${ }^{\circledR}$ (eslicarbazepine acetate) prescribing information. Revised 03/2019.

13. Gil-Nagel A, Lopes-Lima J, Almeida L, et al. Efficacy and safety of 800 and $1200 \mathrm{mg}$ eslicarbazepine acetate as adjunctive treatment in adults with refractory partial-onset seizures. Acta Neurol Scand. 2009;120(5):281-287. doi:10.1111/j.1600-0404.2009. 01218.x 
14. Hufnagel A, Ben-Menachem E, Gabbai AA, et al. Long-term safety and efficacy of eslicarbazepine acetate as adjunctive therapy in the treatment of partial-onset seizures in adults with epilepsy: results of a 1-year open-label extension study. Epilepsy Res. 2013;103(23):262-269. doi:10.1016/j.eplepsyres.2012.07.014

15. Sperling MR, Abou-Khalil B, Harvey J, et al. Eslicarbazepine acetate as adjunctive therapy in patients with uncontrolled partial-onset seizures: results of a Phase III, double-blind, randomized, placebo-controlled trial. Epilepsia. 2015a;56(2):244-253. doi:10.1111/epi.12894

16. Sperling MR, Harvey J, Grinnell T, et al. Efficacy and safety of conversion to monotherapy with eslicarbazepine acetate in adults with uncontrolled partial-onset seizures: a randomized historical-control Phase III study based in North America. Epilepsia. 2015b;56(4):546-555. doi:10.1111/epi.12934

17. Cantu D, Gidal BE, Tosiello R, et al. Safety and tolerability of eslicarbazepine acetate as first adjunctive therapy with levetiracetam or lamotrigine, or as later adjunctive therapy in patients with focal seizures. American Epilepsy Society Annual Meeting; 6-10 December 2019; Baltimore (MD). Abstract 1.427.

18. Pikalov A, Grinnell T, Hixson J, et al. Efficacy of eslicarbazepine acetate as first adjunctive therapy with levetiracetam or lamotrigine, or as later adjunctive therapy in patients with focal seizures. American Epilepsy Society Annual Meeting; 6-10 December 2019; Baltimore (MD). Abstract 1.45.

19. Lloyd-Smith A, Hennessy R, Hegde M, et al. Comparison of levetiracetam versus sodium channel blockers as first line antiepileptic drug in participants with high seizure burden using human epilepsy project data. American Epilepsy Society Annual Meeting; 2-6 December 2016; Houston (TX). Abstract 2.103.

20. Trinka E, Ben-Menachem E, Kowacs PA, et al. Efficacy and safety of eslicarbazepine acetate versus controlled-release carbamazepine monotherapy in newly diagnosed epilepsy: a phase III double-blind, randomized, parallel-group, multicenter study. Epilepsia. 2018;59 (2):479-491. doi:10.1111/epi.13993

21. Toledano R, Jovel CE, Jimenez-Huete A, et al. Efficacy and safety of eslicarbazepine acetate monotherapy for partial-onset seizures: experience from a multicenter, observational study. Epilepsy Behav. 2017;73:173-179. doi:10.1016/j.yebeh.2017.02.028

22. Allers K, Essue BM, Hackett ML, et al. The economic impact of epilepsy: a systematic review. BMC Neurol. 2015;15(1):245-266. doi:10.1186/s12883-015-0494-y

23. Cardarelli WJ, Smith BJ. The burden of epilepsy to patients and payers. Am J Manag Care. 2010;16(12 Suppl):S331-S336

24. Ivanova JI, Birnbaum HG, Kidolezi Y, et al. Economic burden of epilepsy among the privately insured in the US. Pharmacoeconomics. 2010a;28(8):675-685. doi:10.2165/11535570-000000000-00000

25. Ivanova JI, Birnbaum HG, Kidolezi Y, et al. Direct and indirect costs associated with epileptic partial onset seizures among the privately insured in the United States. Epilepsia. 2010b;51(5):838-844. doi:10.1111/j.1528-1167.2009.02422.x

26. Begley CE, Durgin TL. The direct cost of epilepsy in the United States: a systematic review of estimates. Epilepsia. 2015;56 (9):1376-1387. doi:10.1111/epi.13084

ClinicoEconomics and Outcomes Research

\section{Publish your work in this journal}

ClinicoEconomics and Outcomes Research is an international, peerreviewed open-access journal focusing on Health Technology Assessment, Pharmacoeconomics and Outcomes Research in the areas of diagnosis, medical devices, and clinical, surgical and pharmacological intervention. The economic impact of health policy and health systems
27. Faught E, Helmers SL, Begley CE, et al. Newer antiepileptic drug use and other factors decreasing hospital encounters. Epilepsy Behav. 2015;45:169-175. doi:10.1016/j.yebeh.2015.01.039

28. Mehta D, Davis M, Epstein AJ, et al. Impact of early initiation of eslicarbazepine acetate on economic outcomes among patients with focal seizure: results from retrospective database analyses. Neurol Ther. 2020;9(2):585-598. doi:10.1007/s40120-020-00211-6

29. US Department of Health and Human Services. Summary of the HIPAA privacy rule. 2; 2013. Available from: https://www.hhs.gov/ hipaa/for-professionals/privacy/laws-regulations/index.html. Accessed December 3, 2020.

30. US Bureau of Economic Analysis. 'Table 1.1.4. Price Indexes for Gross Domestic Product'. Available from: https://apps.bea.gov/ iTable/iTable.cfm?reqid=19\&step=2. accessed December 3, 2020.

31. Dunn A, Grosse SD, Zuvekas SH. Adjusting health expenditures for inflation: a review of measures for health services research in the United States. Health Serv Res. 2018;53(1):175-196. doi:10.1111/ 1475-6773.12612

32. Linden A, Adams JL. Applying a propensity score-based weighting model to interrupted time series data: improving causal inference in programme evaluation. J Eval Clin Pract. 2011;17(6):1231-1238. doi:10.1111/j.1365-2753.2010.01504.x

33. Austin PC. An introduction to propensity score methods for reducing the effects of confounding in observational studies. Multivariate Behav Res. 2011;46(3):399-424. doi:10.1080/00273171.2011.568786

34. Cameron AC, Miller DL. A practitioner's guide to cluster-robust inference. J Human Res. 2015;50(2):317-372. doi:10.3368/jhr.50.2. 317

35. Stuart EA, Lee BK, Leacy FP. Prognostic score-based balance measures can be a useful diagnostic for propensity score methods in comparative effectiveness research. J Clin Epidemiol. 2013;66(8): SS84-SS90.e1. doi:10.1016/j.jclinepi.2013.01.013

36. Heaney D, Beran R, Halpern M. Economics in epilepsy treatment choices: our certain fate? Epilepsia. 2002;43:32-38. doi:10.1046/ j.1528-1157.43.s.4.6.x

37. French JA, Gazzola DM. New generation antiepileptic drugs: what do they offer in terms of improved tolerability and safety? Ther Adv Drug Saf. 2011;2(4):141-158. doi:10.1177/2042098611411127

38. Mäkinen J, Rainesalo S, Peltola J. Transition from oxcarbazepine to eslicarbazepine acetate: a single center study. Brain Behav. 2017;7 (3):e00634. doi:10.1002/brb3.634

39. Jalihal V, Shankar R, Henley W, et al. Eslicarbazepine acetate as a replacement for levetiracetam in people with epilepsy developing behavioral adverse events. Epilepsy Behav. 2018;80:365-369. doi:10.1016/j.yebeh.2018.01.020

40. Kim SH, Lee H, Kim DW. Switching antiepileptic drugs to once-daily dosing regimens in epilepsy patients. Acta Neurol Scand. 2021;143(1):51-55. doi:10.1111/ane.13333

41. Ferrari CM, de Sousa RM, Castro LH. Factors associated with treatment non-adherence in patients with epilepsy in Brazil. Seizure. 2013;22(5):384-389. doi:10.1016/j.seizure.2013.02.006

organization also constitute important areas of coverage. The manuscript management system is completely online and includes a very quick and fair peer-review system, which is all easy to use. Visit http://www.dovepress.com/testimonials.php to read real quotes from published authors. 